\title{
High level expression of bikunin in Pichia pastoris by fusion of human serum albumin
}

\author{
Xing-Hua Gou', Yu-Ying Liu², Qi-Lei Chen', Jian-Jun Tang², Da-Yu Liu', Liang Zou', Xiao-Yong Wu' and \\ Wei Wang ${ }^{1,3^{*}}$
}

\begin{abstract}
Bikunin is a proteoglycan exhibiting broad-spectrum inhibitory activity against serine proteases and could potentially suppress tumor cell invasion and metastasis. Here, we have successfully expressed recombinant human bikunin (rh-bikunin) in Pichia pastoris and also established the purification procedure. Different fusion genes of hUTI and domain I, domain I and domain II, domain I, domain II and domain III of human serum albumin (HSA) were inserted into expression vector pPICZ $\alpha$ A. After expressed in shake flask, rh-bikunin was produced in an 30-L fermenter and purified by affinity chromatography and cation exchange chromatography. The final expression levels were $200 \mathrm{mg} / \mathrm{L}$ and we got totally $1.08 \mathrm{~g}(3650 \mathrm{lU} / \mathrm{mg}$ ) of active purified rh-bikunin (purity is 98\%) from $20 \mathrm{~L}$ of fermentation broth. The rh-bikunin consists of unique form with molecular masses of $25 \mathrm{kDa}$, and has the same $\mathrm{N}$-terminals sequence as human native bikunin. This study provided a new method for high level expression of active rh-bikunin by using HSA as fusion parter.
\end{abstract}

Keywords: Human bikunin, Fusion expression, Human serum albumin, Pichia pastoris

\section{Introduction}

Bikunin, also being called urinary trypsin inhibitor (UTI), contains two antiproteolytic Kunitz domains. The protein is a proteoglycan ([Xu, Carr et al. 1998]), which has a molecular mass of about $25 \mathrm{kDa}$ including a 6-7 $\mathrm{kDa}$ chondroitin sulfate chain ([Pugia, Valdes Jr et al. 2007]; [Chi, Wolff et al. 2008]). Bikunin is synthesized in the liver together with another plasma protein, $\alpha_{1-}$ microglobulin $\left(\alpha_{1}-\mathrm{m}\right)$, forming a precursor $\left(\alpha_{1}-\mathrm{m} / \mathrm{biku}\right.$ nin precursor, $A M B P$ ). As a kind of serine proteinase inhibitor, bikunin exhibits broad inhibitory activity against many proteases, such as trypsinase, chymotrypsin, leukocyte elastase, and fibrinolytic enzyme. Moreover, human bikunin hasn't antigenicity to human and has the characteristic of use safety, so it has been widely used as a drug for patients with acute pancreatitis, acute attack of chronic pancreatitis, acute circulation exhaustion, tumor and shock ([H Inaba 1986]; [Okuhama, Shiraishi et al. 1999]; [Kobayashi, Suzuki et al. 2003]; [Yano, Anraku et al. 2003]; [Molor-Erdene, Okajima et al.

\footnotetext{
* Correspondence: wangwei@cdu.edu.cn

'Faculty of Bioindustry, Chengdu University, Waidong Shilingzhen, Chengdu, 610106, China

Full list of author information is available at the end of the article
}

2005]; [Qing xia 2005]; [Zhang, Liu et al. 2011]). The bikunin has many advantages such as evident effect in clinic, low side effect and low production cost. However, due to the low content in urinary, difficult collection of human urinary and high cost of purification, the bikunin is limited to apply widely. To overcome these problems, a promising alternative technique is to obtain recombinant human bikunin by gene recombination.

The bikunin have been successfully cloned and expressed in E. coli and Pichia pastoris ([Fritz 1995]; [Brinkmann, Weilke et al. 1997]; [Jian-qiu, Feng-qin et al. 2008]). However, the yield of recombinant human UTI (rh-UTI) in E. coli or P. pastoris is too low and the uniform of protein doesn't to be ensured. There hasn't been report about large scale production and animal model examination so far. Therefore, the clinic value of rh-UTI is difficult to be determined all the same.

Previous study showed that the use of human serum albumin (HAS) as N-terminal fusions can be an effective technique to express difficult proteins in mammalian cells ([Carter, Zhang et al. 2010]; [Zhang, Carter et al. 2010]). So in this study, fusion genes of h-UTI and domain I, domain I and domain II, domain I, domain II and domain III of human serum albumin were inserted 
into expression vector pPICZ $\alpha \mathrm{A}$, respectively. Finally, all plasmids were linearized for transformation into $P$. pastoris strain GS115. The h-UTI was highly expressed in $P$. pastoris, which successfully solved the problems of the uniform and low yield of h-UTI expressed in $P$. pastoris.

\section{Materials and methods}

\section{Strains, vectors and other reagents}

The $P$. pastoris GS115, pPICZ $\alpha$ A vector, and Zeocin antibiotic were obtained from Invitrogen (CA, USA). $P$. pastoris were grown in YPD medium containing $10 \mathrm{~g} / \mathrm{L}$ yeast extract, $20 \mathrm{~g} / \mathrm{L}$ peptone, and $20 \mathrm{~g} / \mathrm{L}$ D-glucose. To prepare YPD plates, $2 \%$ agar $(\mathrm{w} / \mathrm{v})$ was added to the YPD medium. YPD-Zeocin plates (1\% yeast extract, $2 \%$ peptone, $2 \%$ dextrose, $2 \%$ agar, and 0.1-0.2 $\mathrm{mg} / \mathrm{mL}$ Zeocin) were used for selecting multicopy transformants. The $P$. pastoris cells were cultured in BMGY medium (1\% yeast extract, $2 \%$ peptone, $1 \%$ glycerol, $400 \mu \mathrm{g} / \mathrm{L}$ biotin, and $0.1 \mathrm{M}$ potassium phosphate, $\mathrm{pH}$ 6.0) for growth and in BMMY medium (1\% yeast extract, 2\% peptone, $400 \mu \mathrm{g} / \mathrm{L}$ biotin, $1 \%$ methanol, and $0.1 \mathrm{M}$ potassium phosphate, $\mathrm{pH}$ 6.0) for induction. All primers were synthesized by Sangon Biotechnology Corp. (Shanghai, China). All restriction enzymes, DNA marker, synthesized genes (human BSA-UTI fusions) and protein marker were purchased from Takara (Dalian, China). The standard human UTI, trypsin purchased from Sigma-Aldrich (St. Louis, USA).

\section{Construction of expression vector pPICZ $\alpha$-HSA-UTI}

Construction of rh-bikunin expression vector was based on $\mathrm{pPICZ} \alpha \mathrm{A}$ vector. The synthesized human serum albumin (HSA) gene containing different domains were added $6 \times$ His tag, several Gly linkers and the recognition site of Enterokinase (EK) at the C-terminal (as shown in Figure 1). The synthesized HSA gene and UTI gene were ligated to $\mathrm{pMD} 18-\mathrm{T}$ vector. The recombinant plasmids pMD-HSA-UTI were digested with Xho I and Not I and then inserted into the same site of pPICZ $\alpha$ A. The recombinant expression plasmids were designated as pPICZ $\alpha$-UTI, pPICZ $\alpha$-D1-UTI, pPICZ $\alpha$-D2-UTI, pPICZ $\alpha-D 12-U T I$ and pPICZ $\alpha-D 123-U T I$, respectively.

\section{Transformation of $P$. pastoris and selection of high-level expression}

The five Sac I restriction endonuclease linearized recombinant expression vectors were introduced into $P$. pastoris GS115 by electroporation using a Micropulser (Bio-Rad, USA) according to the manual, respectively.

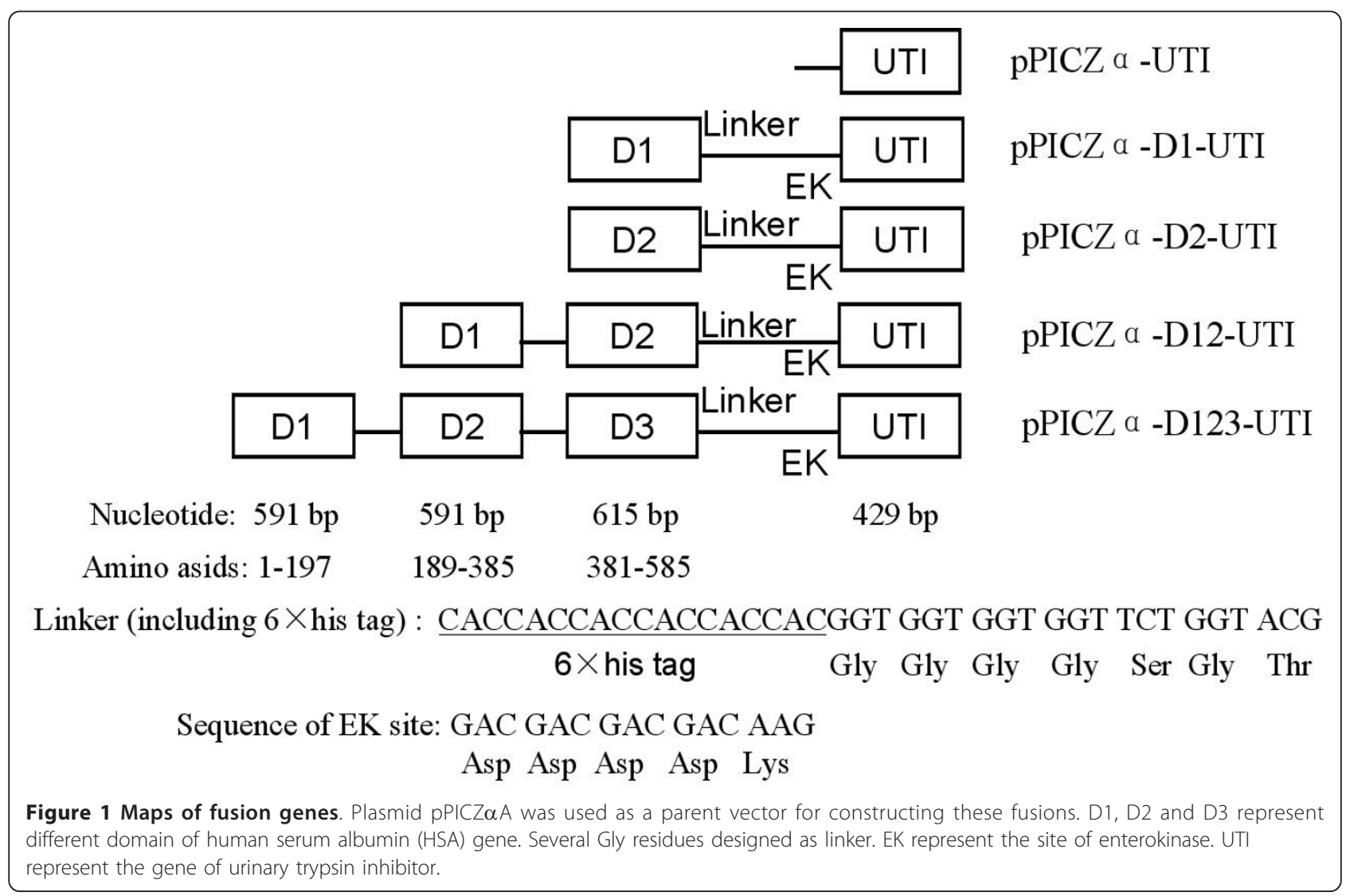


Transformed cells were selected by growth on yeast extract peptone dextrose (YPD) agar plates containing Zeocin $(0.5 \mathrm{mg} / \mathrm{mL})$. After the multicopy transformants appeared, single clone was cultured in $5 \mathrm{~mL}$ BMGY medium at $28^{\circ} \mathrm{C}$ with shaking at $250 \mathrm{rpm}$ for $24 \mathrm{~h}$. The cells were then centrifuged and resuspended in $5 \mathrm{~mL}$ BMMY medium to induce expression for 4 days. The culture medium $(0.5 \mathrm{~mL})$ was sampled per day and centrifuged at $4{ }^{\circ} \mathrm{C}, 10,000 \mathrm{rpm}$ for $5 \mathrm{~min}$. Cell pellet and supernatant were separated. The supernatant was tested for UTI activity and cell pellet was used for genomic DNA analysis. pPICZ $\alpha$ A blank plasmids were also transformed as a negative control.

\section{Optimization of fermention conditions by shake-flask mode}

In order to determine the effect of $\mathrm{pH}$ on the expression level of rh-UTI, the BMGY media was performed at different $\mathrm{pH}$ values $(\mathrm{pH} 4.0,5.0,5.5,6.0,6.5,7.0)$. In order to determine the effect of feeding mode on the expression level of rh-UTI, inorganic salt, peptone, yeast extracts and glycerol was used as the carbon sources in BMGY media by continuous fed-batch mode, respectively.

\section{High-density fermentation}

A stock culture of $P$. pastoris was grown to an $\mathrm{A}_{600}$ of 3-6 in a 5-L shake flask containing $2 \mathrm{~L}$ YPD. The shake flask culture was used to inoculate an 30-L fermenter (Bioengineering, AG) containing $20 \mathrm{~L}$ of fermentation basal salts medium FM22 supplemented with PTM1 trace salts $(1.1 \mathrm{~mL}$ of stock solution/L) and biotin $(0.4$ $\mathrm{mL}$ of the stock solution/L). The dissolved oxygen level (DO) was set at $30 \%$ and the stirring rate was $700 \mathrm{rpm}$. The $\mathrm{pH}$ of the medium was maintained 6.0-6.5 by automatic addition of $5 \mathrm{~N} \mathrm{NH}_{4} \mathrm{OH}$ and $1 \mathrm{M}$ phosphoric acid and $5 \%$ antifoam as required. Temperature was maintained at $30^{\circ} \mathrm{C}$. The initial cultivation terminated when all glycerol was consumed (about $14 \mathrm{~h}$ ) at batch phase. Continuous $2 \%$ peptone and $1 \%$ yeast extract feeding were carried out for about $6 \mathrm{~h}$ in the subsequent fedbatch phase. To induce rh-UBI expression, 100\% methanol was fed at $3 \mathrm{~mL} / \mathrm{h} / \mathrm{L}$ for $50 \mathrm{~h}$. Sampling of the culture medium at the end of each phase was performed for assay of rh-bikunin activity.

\section{Purification of rh-UBI and assay of trypsin inhibitory activity}

The supernatant was collected by centrifugation, $5 \mathrm{M}$ $\mathrm{NaCl}$ and phosphate buffer was added to a final concentration of $1 \mathrm{M}$ and $50 \mathrm{mM}$, respectively. Finally, the $\mathrm{pH}$ value was also adjiusted to 7.4 with $\mathrm{NaOH}$. Then the treated supernatant was was clarified with a $0.45 \mu \mathrm{m}$ cellulose membrane. The supernatant was purified with chelating sepharose affinity chromatography and Q sepharose F. F. anion exchange chromatography in turn. Then after digested by enterokinase, the sample was isolated and purified by chelating sepharose affinity chromatography and SP sepharose F.F cation exchange chromatography. The purity of each step protein sample was checked by SDS-PAGE. The final rh-bikunin protein was stored at $-20^{\circ} \mathrm{C}$ for further assay of trypsin inhibitory activity accroding to previous study ([Page, Quillien et al. 2000]; [Wang, Yan et al. 2008]).

\section{Carbohydrate digestion and amino-terminal sequence}

The amino-terminal sequence of rh-bikunin was determined automated Edman degradation method (Shanghai Sangon Biological Engineering Technology \& Services CO., Ltd). N-linked glycosylation was assayeded by digestion of the protein with $\mathrm{N}$-glycosidase F. Protein samples $(20 \mu \mathrm{g})$ were boiled for $5 \mathrm{~min}$ in $1 \%(\mathrm{w} / \mathrm{v})$ SDS and $50 \mathrm{mM}$ DTT. Samples were then diluted to $0.1 \%$ SDS, $5 \mathrm{mM}$ DTT, $20 \mathrm{mM}$ sodium phosphate ( $\mathrm{pH} 7.4$ ), $25 \mathrm{mM}$ EDTA, and $2 \%$ Triton X-100. N-glycosidase F $(0.8 \mathrm{U})$ was added and samples were incubated at $37^{\circ} \mathrm{C}$ overnight. Deglycosylation of the protein was assessed by a shift in electrophoretic mobility on SDS-PAGE analysis.

\section{Results}

\section{Construction of recombinant plasmids and identification} of transformed colonies

Five plasimds containing of different domain of HSA gene were constructed (Figure 1). DNA sequence analysis of the five recombinant expression vectors $\mathrm{pPICZ} \alpha$ HSA-UTI demonstrated that cDNA encoding human bikunin and human serum albumin (HSA) gene were correctly inserted into pPICZ $\alpha$ A vector. The recombinant expression vectors were then linearized with Sac I and introduced into P. pastoris strain GS115. Agarose gel electrophoresis of the PCR products showed that cDNA encoding bikunin was indeed integrated into the the $P$. pastoris genome. However, there were no visible bands from the control sample which were transformed with pPICZaA blank plasmid.

\section{Shake-flask expression of rh-bikunin}

Three clonies containing of pPICZ $\alpha$-D12-UTI showed the highest expression level in shake-flask expression than other recombinant plasmids (Data not shown). The effects of $\mathrm{pH}$ value and carbon sources on the expression level of rh-UTI in P. pastoris/pPICZ $\alpha$-D12-UTI were also investigated by shake-flask fermentation. The $\mathrm{pH}$ value of BMGY media was adjusted to the desired value. The results showed that the expression levels of rh-bikunin were different when $\mathrm{pH}$ of medium varied from 4.0-7.0. When the $\mathrm{pH}$ value was 6.0-6.5, the 
expression level of rh-UBI was higher than those of other $\mathrm{pH}$ values. In order to determine the effect of feeding mode on the expression level of rh-UBI, inorganic salt, peptone, yeast extracts and glycerol was used as the carbon sources in BMGY media by continuous fed-batch mode, respectively. The results displayed that when the inorganic salt, peptone and yeast extracts were used as the carbon sources in BMGY media, the expression level of rh-UBI was higher than that of glycerol. Therefore, the optimal $\mathrm{pH}(6.0-6.5)$ and carbon sources (inorganic salt, peptone or yeast extracts) were selected as shake-flask condition and further fermentation (data not shown).

\section{High-density fermentation and purification of the recombinant protein}

Under optimal conditions $\left(\mathrm{pH} 6.0,30^{\circ} \mathrm{C}\right.$, and $\mathrm{DO}$ set at about $30 \%$ ), the highest optical density and cell wet weight were obtained after $50 \mathrm{~h}$ of methanol induction. The expression level was also reached to $200 \mathrm{mg} / \mathrm{L}$. The rh-bikunin solution was purified with chelating sepharose affinity chromatography and Q sepharose F.F. anion exchange chromatography in turn. Then after digested by enterokinase, the sample was isolated and purified by chelating sepharose affinity chromatography and SP sepharose F.F cation exchange chromatography. The protein recovery of the rh-bikunin at the different purification steps were summarized in Table 1. Following these processes, we could get totally $1.08 \mathrm{~g}$ of purified rh-bikunin (purity is $98 \%$ ) from $20 \mathrm{~L}$ culture medium as revealed by SDS-PAGE (Figure 2 and Table 1). Rh-bikunin was secreted in the medium after induction and a final concentration of approximately $3650 \mathrm{IU} / \mathrm{mg}$ was obtained, which was 1.46 times higher than that of native human urinary bikunin (2500 IU/mg).

\section{Characterization of purified rh-UTI}

According to SDS-PAGE analysis (Figure 2, Lanes S3 and S4), rh-UTI migrated as a single band. The molecular masses based on protein migration rates in SDS gel were $23 \sim 24 \mathrm{kDa}$. However, double band was observed in other previous study ([Falkenberg, Wester et al.
2001]; [Wang, Yan et al. 2008]). The mass of rh-UTI increased by approximately $5 \mathrm{kDa}$ after treatment with $\mathrm{N}$-glycosidase F (Figure 3, Lanes 3, 4 and 5). On the other hand, the $\mathrm{N}$-terminal amino acid sequence of rhUTI was also identical to the $\mathrm{N}$-terminus of native human bikunin (Figure 4, Ala-Val-Leu-Pro), which demonstrating correct processing ([Kakizaki, Takahashi et al. 2007]).

\section{Discussion}

The expression system of yeast $P$. pastoris has several advantages, including the use of the alcohol oxidase I (AOX1) gene promoter, the ability of the cells to be cultivated at high density, a simplified purification procedure for secreted heterologous proteins, and modifications of foreign proteins ([Wang, Yan et al. 2008]). So, the expression system of yeast $P$. pastoris was used for bikunin expression. On the other hand, several parameters such as clone selection, codon optimization, fusion partners and culture conditions play a important role in obtaining high yields of protein. So, in order to optimize the level of protein production experiment, we evaluated the role of codon optimization, fusion partners and culture conditions in obtaining high yields of bikunin.

Firstly, codon bias is an intrinsic problem in heterologous protein production and needs to be taken into account in the experimental design. So, the synthesized human serum albumin (HSA) gene was used in this study. Secondly, previous study showed that the use of human serum albumin (HAS) as N-terminal fusions can be an effective technique to express difficult proteins in mammalian cells ([Carter, Zhang et al. 2010]; [Zhang, Carter et al. 2010]). So in this study, fusion genes of hUTI and domain I, domain I and domain II, domain I, domain II and domain III of human serum albumin were inserted into expression vector pPICZ $\alpha \mathrm{A}$, respectively (As shown in Figure 1). The results showed that three clonies containing of pPICZ $\alpha$-D12-UTI (containing domain I and domain II) showed the highest expression level in shake-flask expression than other recombinant plasmids. Thirdly, the effects of $\mathrm{pH}$ value

Table 1 Summary of purification process of rh-UTI

\begin{tabular}{|c|c|c|c|c|}
\hline Purification step & $\begin{array}{l}\text { Volume } \\
\text { (mL) }\end{array}$ & $\begin{array}{c}\text { Protein concentration } \\
\text { (mg/L) }\end{array}$ & $\begin{array}{c}\text { rh-UTI } \\
\text { (mg) }\end{array}$ & Protein recovery (\%) \\
\hline Supernatant & 20,000 & 200 & 4,000 & \\
\hline Ultrafiltrated & 3,000 & 1.20 & 3,600 & 90.0 \\
\hline Chelating sepharose & 600 & 4.75 & 2,850 & 71.25 \\
\hline Q sepharose F.F. & 350 & 6.68 & 2,338 & 58.45 \\
\hline Chelating sepharose after digested by Enterokinase & 1,500 & 0.90 & 1,350 & 33.75 \\
\hline SP sepharose F.F & 220 & 4.91 & 1,080 & 27.0 \\
\hline
\end{tabular}




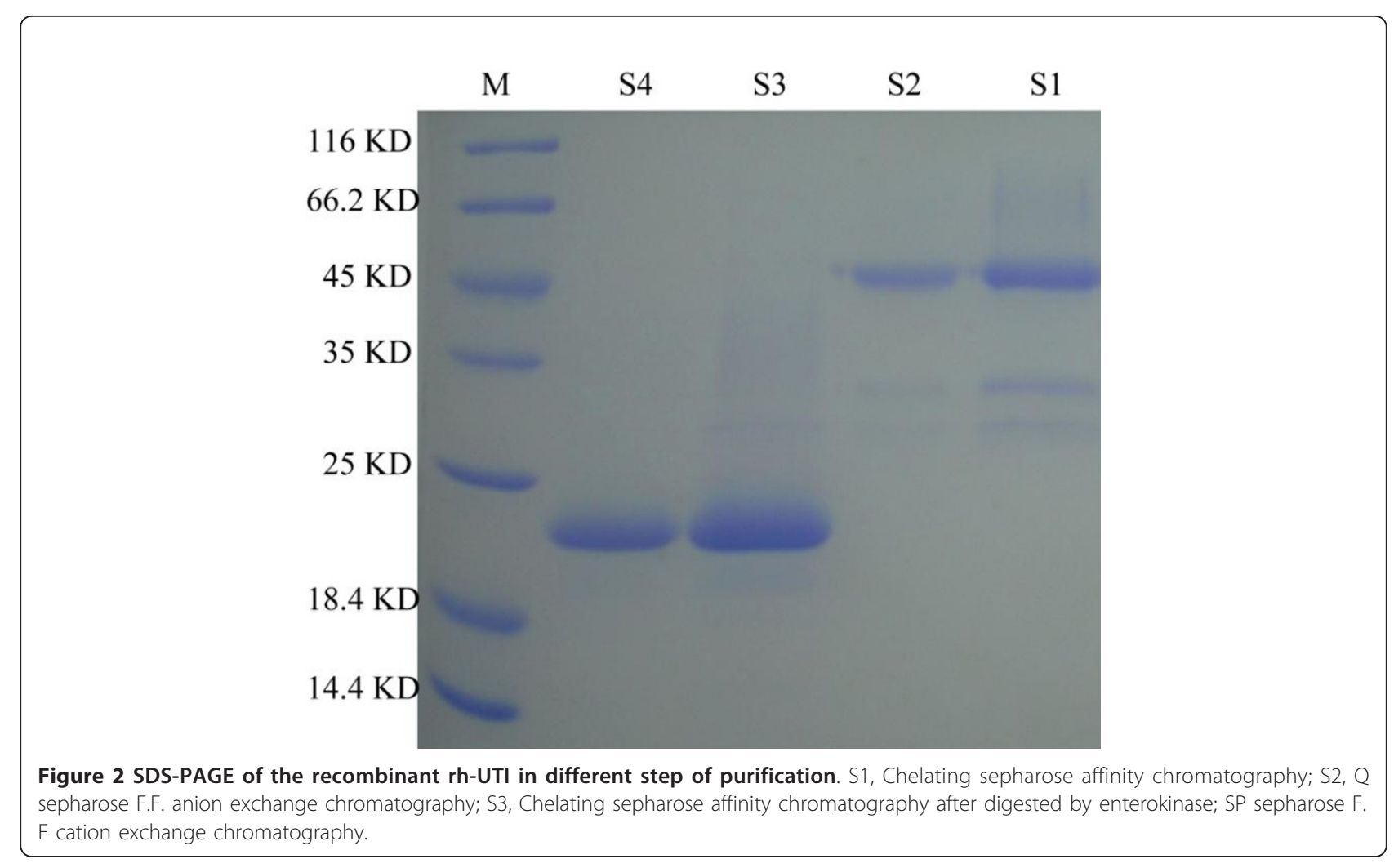

and carbon sources on the expression level of rh-UTI in P. pastoris/pPICZ $\alpha$-D12-UTI were also investigated by shake-flask fermentation. Rh-bikunin was secreted in the medium after induction and the final expression levels were $200 \mathrm{mg} / \mathrm{L}$. As a result, we got totally $1.08 \mathrm{~g}(3650$ $\mathrm{IU} / \mathrm{mg}$ ) of active purified rh-bikunin (purity is 98\%) from $20 \mathrm{~L}$ of fermentation broth, which was 1.46 times higher than that of native human urinary bikunin $(2500$

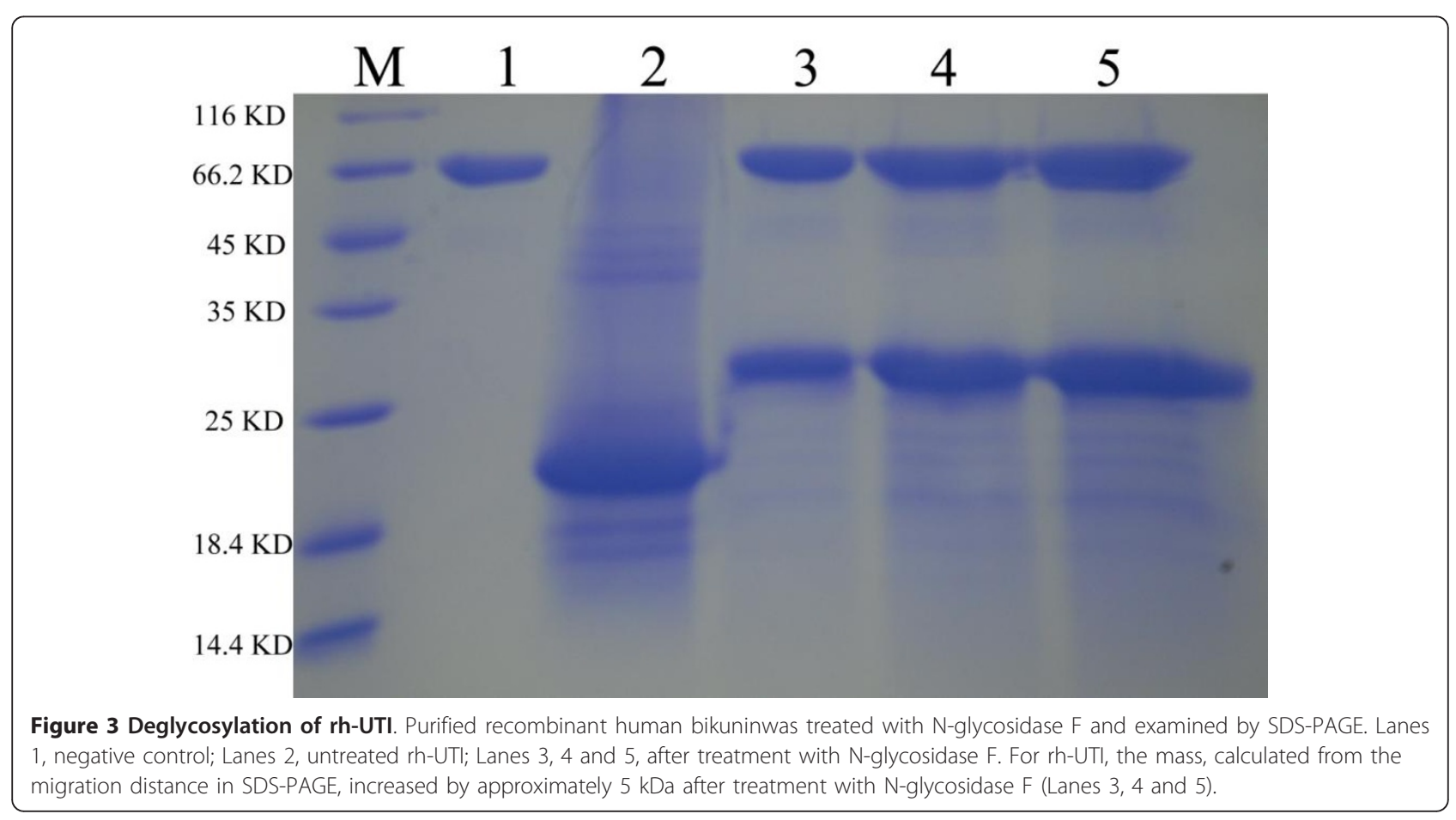



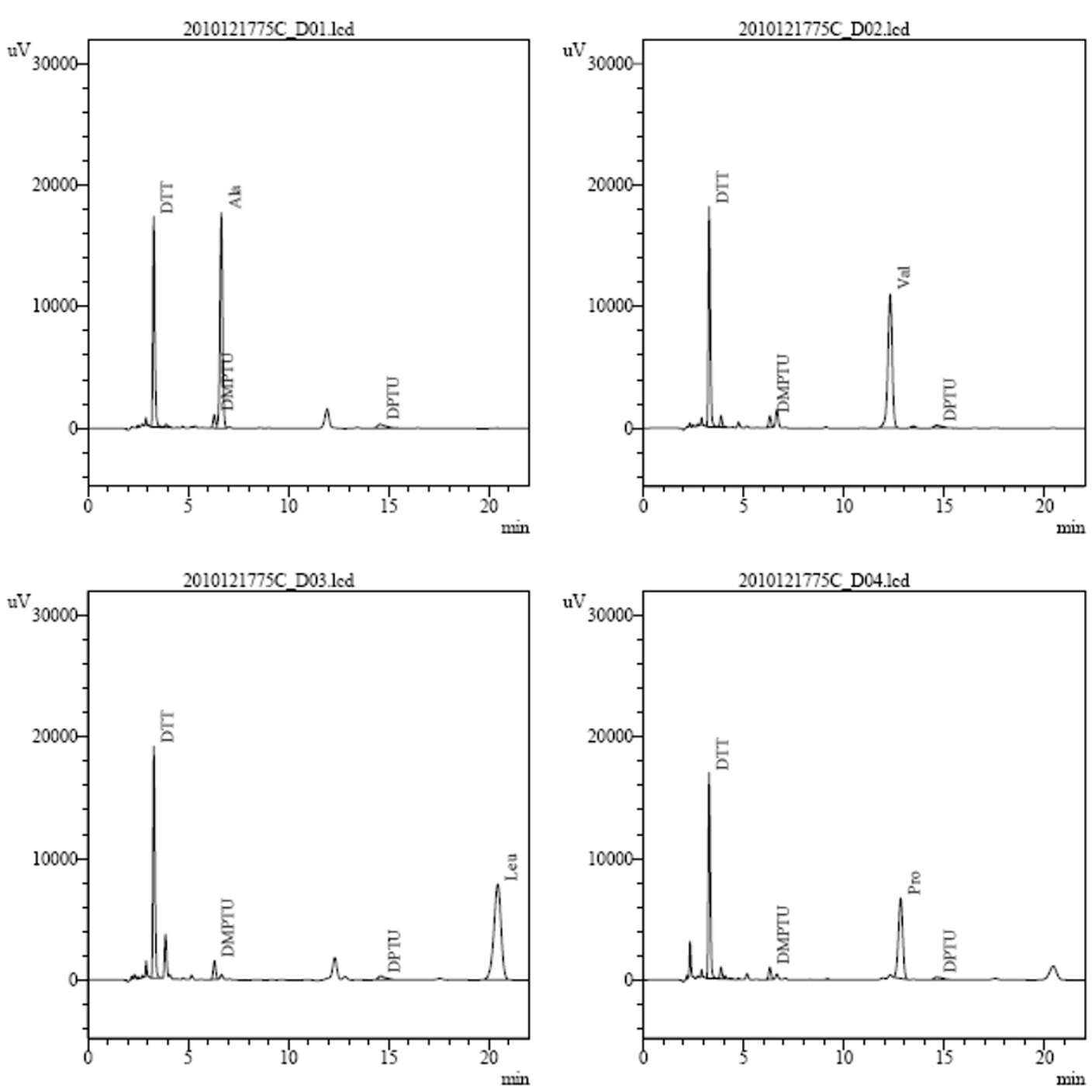

Figure 4 Amino-terminal sequence of rh-UTI. N-terminus of rh-bikunin was Ala-Val-Leu-Pro.

IU/mg) ([Wang, Yan et al. 2008]). By combined these methods, we sucessfully expressed active rh-bikunin at the high level.

\section{Acknowledgements}

This work was kindly supported by the Applied \& Basic Research Foundation of Chengdu City Science and Technology Bureau, Chengdu, China (No: 10GGYB331SW-023)

\section{Author details}

${ }^{1}$ Faculty of Bioindustry, Chengdu University, Waidong Shilingzhen, Chengdu, 610106, China ${ }^{2}$ Genekey Biotech. (Chengdu) Co., Ltd. Chengdu, 610041

China ${ }^{3}$ Meat Proceeding Key Lab. of Sichuan Province, 610106, China

\section{Competing interests}

The authors declare that they have no competing interests.

Received: 21 December 2011 Accepted: 29 February 2012

Published: 29 February 2012

\section{References}

Brinkmann T, Weilke C (1997) Recognition of Acceptor Proteins by UDP-D-xylose Proteoglycan Core Protein beta-D-Xylosyltransferase. J Biol Chem 272:11171-11175. doi:10.1074/jbc.272.17.11171.

Carter J, Zhang J, Dang TL, Hasegawa H, Cheng JD, Gianan I, O'Neill JW, Wolfson M, Siu S, Qu S, Meininger D, Kim H, Delaney J, Mehlin C (2010) Fusion partners can increase the expression of recombinant interleukins via transient transfection in 2936E cells. Protein Sci 19:357-362. doi:10.1002/ pro.307.

Chi L, Wolff JJ, Laremore TN (2008) Structural Analysis of Bikunin Glycosaminoglycan. J Am Chem Soc 130:2617-2625. doi:10.1021/ja0778500.

Falkenberg C, Wester L (2001) Expression of a functional proteinase inhibitor capable of accepting xylose: bikunin. Arch Biochem Biophys 387:99-106. doi:10.1006/abbi.2000.2213.

Fritz H, Gebhard W, Rathindra D (1995) Human Bikunin variants as proteinase inhibitors, and medicaments containing these. US Patent 5,407,915

Inaba H, Kato K, Ohzawa N, Nakayama K (1986) Effect of urinastatin on disseminated intravascular coagulation. Nippon Yakurigaku Zasshi 88:239-244. doi:10.1254/fpj.88.239. 
Wang JQ, Yan FQ, Wang DD, Ban L, Sun N, Li CY, Zhang T, Yan WQ (2008) Expression and purification of active recombinant human bikunin in Pichia pastoris. Protein Expr Purif 60:127-131. doi:10.1016/j.pep.2008.03.025.

Kakizaki I, Takahashi R, Ibori N (2007) Diversity in the degree of sulfation and chain length of the glycosaminoglycan moiety of urinary trypsin inhibitor isomers. Biochim Biophys Acta 1770:171-177. doi:10.1016/j. bbagen.2006.09.026.

Kobayashi H, Suzuki M, Hirashima Y, Terao T (2003) The protease inhibitor bikunin, a novel anti-metastatic agent. Biol Chem 384:749-754. doi:10.1515/ BC.2003.083.

Molor-Erdene P, Okajima K, Isobe H, Uchiba M, Harada N, Okabe H (2005) Urinary trypsin inhibitor reduces LPS-induced hypotension by suppressing tumor necrosis factor-alpha production through inhibition of Egr-1 expression. Am J Physiol Heart Circ Physiol 288:1265-1271

Okuhama Yukihiro, Shiraishi Masayuki, Higa Takao, Tomori Hirofumi, Taira Kaoru, Mamadi Toure, Muto Yoshihiro (1999) Protective effects of ulinastatin against ischemia-reperfusion injury. J Surg Res 82:34-42. doi:10.1006/jsre.1998.5496.

Page D, Quillien L (2000) Trypsin inhibitory activity measurement. Crop Sci 40:1482-1485. doi:10.2135/cropsci2000.4051482x.

Pugia MJ, Valdes JR, Jortani SA (2007) Bikunin (Urinary Trypsin Inhibitor): Structure, Biological Relevance, And Measurement. Adv Clin Chem 44:223-245

Xia Qing, Yuan Lin, Yang Xiao-Nan, Tang Wen-Fu, Jiang Jun-Ming (2005) Comparison of integrated Chinese and Western medicine with and without somatostatin supplement in the treatment of severe acute pancreatitis. World J Gastroenterol 11:1073-1076

Wang Jian-Qiu, Yan Feng-Qin, Wang Dou-Dou, Ban Lei, Sun Nan, Li Cong-Yan, Zhang Ting, Yan Wei-Qun (2008) Expression and purification of active recombinant human bikunin in Pichia pastoris. Protein Expr Purif 60:127-131. doi:10.1016/j.pep.2008.03.025.

Yi-bin Xu, Carr PD, Guss JM, David LO (1998) The crystal structure of bikunin from the inter-alpha-inhibitor complex: a serine protease inhibitor with two Kunitz domains. J Mol Biol 276:955-966. doi:10.1006/jmbi.1997.1582.

Yano, Toshiyuki, Anraku, Sakiko, Nakayama, Ryosuke, Ushijima, Kazuo (2003) Neuroprotective effect of urinary trypsin inhibitor against focal cerebral ischemia-reperfusion injury in rats. Anesthesiology 98:465-473. doi:10.1097/ 00000542-200302000-00028.

Zhang J, Carter J, Siu S, O'Neill JW, Gates AH, Delaney J, Mehlin C (2010) Fusion partners as a tool for the expression of difficult proteins in mammalian cells. Curr Pharm Biotechnol 11:241-245. doi:10.2174/138920110791111898.

Zhang XY, Liu FQ, Liu HY, Cheng HX, Wang W, Wen Q, Wang YL (2011) Urinary trypsin inhibitor attenuates lipopolysaccharide-induced acute lung injury by blocking the activation of p38 mitogen-activated protein kinase. J Inflamm Res 60:569-575. doi:10.1007/s00011-010-0305-2.

doi:10.1186/2191-0855-2-14

Cite this article as: Gou et al.: High level expression of bikunin in Pichia pastoris by fusion of human serum albumin. AMB Express 2012 2:14.

\section{Submit your manuscript to a SpringerOpen ${ }^{\mathcal{O}}$ journal and benefit from:}

- Convenient online submission

- Rigorous peer review

- Immediate publication on acceptance

- Open access: articles freely available online

- High visibility within the field

- Retaining the copyright to your article

Submit your next manuscript at $\gg$ springeropen.com 